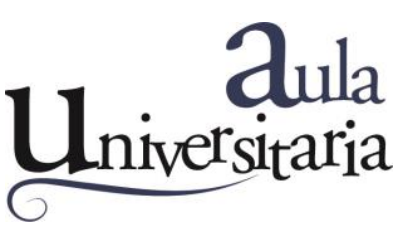

\title{
Promoviendo la inclusión educativa a través de la educación alimentaria nutricional
}

\section{El caso de una escuela de Jóvenes-Adultos de la ciudad de Santa Fe}

\author{
PARAVANO, Antonella; ${ }^{1}$ ORTIGOZA, Liliana Del V.:2 DEZAR, Gimena V. ${ }^{3}$
}

\author{
Filiaciones institucionales \\ ${ }^{1}$ Graduada de la Facultad de Bioquímica y Ciencias Biológicas. Universidad Nacional del Litoral. \\ ${ }^{2}$ Docente del Departamento de Física. Facultad de Bioquímica y Ciencias Biológicas. Universidad Nacio- \\ nal del Litoral. \\ ${ }^{3}$ Directora de Extensión Social y Cultural. Docente de la cátedra de Epidemiología y Salud Pública. Facul- \\ tad de Bioquímica y Ciencias Biológicas. Universidad Nacional del Litoral.
}

\section{Correspondencia}

Facultad de Bioquímica y Ciencias Biológicas. Universidad Nacional del Litoral. Ciudad UniversitariaParaje El Pozo. Ruta Nacional 168. CC 242. Santa Fe. Argentina. Tel: 54-342-4575215/16.

mparavano@rectorado.unl.edu.ar | gdezar@unl.edu.ar | liliortigoza@gmail.com

\section{Resumen}

La Educación Alimentaria Nutricional (EAN) y su influencia en la salud aportan contenidos y competencias a trabajar en la escuela con el objetivo de mejorar la conducta alimentaria y elevar la calidad de vida. Cuando se combinan la EAN y la alimentación escolar puede mejorar directamente la salud y la nutrición de los estudiantes, al tiempo que les ayuda a desarrollar buenos hábitos alimenticios.

Lo mencionado anteriormente puede aplicarse en todas las fases de la vida, teniendo especial importancia en la niñez, adolescencia y en jóvenes-adultos. Promover conductas alimentarias favorables para la salud y un estilo de vida saludable es primordial para conseguir una mejor calidad de vida.

El presente trabajo relata la experiencia realizada en una Escuela de Enseñanza Media para Adultos de la ciudad de Santa Fe, con 100 alumnos que participaron voluntariamente y que se encontraban cursando el tercer y quinto año durante 2016. Se realizó 
un estudio de tipo transversal, descriptivo e interpretativo. Se diseñaron e implementaron talleres lúdico-didácticos durante tres semanas consecutivas. Posteriormente, se aplicó una encuesta de opinión a los participantes de los talleres con el fin de valorar los mismos. Los alumnos participaron de manera activa en cada uno de los encuentros, hubo gran de interés y motivación. Mediante el uso de estrategias lúdico-didácticas e innovadoras fue posible fomentar hábitos saludables, lo que impactó positivamente en el grado de conocimiento sobre un estilo de vida saludable de esta población.

\section{Palabras clave}

educación alimentaria nutricional | jóvenes-adultos | hábitos alimentarios.

\section{Summary}

Nutritional Food Education (EAN) and its influence on health contribute content and skills to work in school, with the aim of improving eating behavior and raising the quality of life. When combining EAN and school feeding, it can directly improve the health and nutrition of students, while helping them develop good eating habits.

The aforementioned can be applied in all phases of life, having special importance in childhood, adolescence and young adults. Promoting healthy eating behaviors and a healthy lifestyle is essential to achieve a better quality of life.

The present work recounts the experience carried out in a Middle School for Adults in the city of Santa Fe, with 100 students who voluntarily participated and were in the third and fifth year, during 2016. A cross-sectional study was carried out., descriptive and interpretive. Fun and educational workshops were designed and implemented for three consecutive weeks. Subsequently, an opinion survey was applied to the workshop participants in order to assess them.

The students participated actively in each of the meetings, there was great interest and motivation. Through the use of playful and innovative strategies, it was possible to promote healthy habits, positively impacting the degree of knowledge about a healthy lifestyle of this population.

\section{Keywords}

Nutritional Food Education | young adult | eating habits

\section{Introducción}

Dentro de la educación para la salud se encuentra el campo de la educación nutricional, cuyo fin es promover la modificación de la conducta alimentaria de la sociedad hacia patrones más saludables. Para ello es imprescindible el desarrollo de programas de educación nutricional cuyo objetivo es la promoción de la salud mediante el aprendizaje, adecuación y aceptación de hábitos alimentarios saludables, de acuerdo con la propia cultura alimentaria y los conocimientos científicos en materia de nutrición que se tienen hasta el momento (Gil Hernández, 2010). 
La FAO (Food and Agriculture Organization) reconoce que es una prioridad realizar intervenciones de nutrición en el ámbito de las instituciones educativas y considera a la escuela como un lugar ideal para la enseñanza de conocimientos básicos en alimentación, nutrición y salud.

En esta dirección, las escuelas educan formando hábitos alimentarios saludables, también llegan a las familias, a la comunidad escolar y pueden ser un canal para la participación de la comunidad en general.

Cuando se combinan la Educación Alimentaria Nutricional (EAN) y la alimentación escolar puede mejorar directamente la salud y la nutrición de los estudiantes, al tiempo que les ayuda a desarrollar buenos hábitos alimenticios.

Promover la nutrición a través de las escuelas puede crear beneficios que se extienden más allá de las aulas y el recreo, para mejorar la salud y el bienestar nutricional de hogares y comunidades (FAO, 2017).

Desde la perspectiva de educación para la salud, se encuentra relevante la definición de EAN como:

\section{La combinación de estrategias educativas, acompañadas de ambientes favorables, diseñadas para facilitar la adopción voluntaria de conductas, comportamientos y elecciones adecuadas en alimentación y nutrición para mantener o mejorar la salud y el bienestar. (Contento, 2011)}

Esta definición atiende al enfoque tridimensional de la nutrición, que distingue al ser humano y a sus problemáticas de salud desde una perspectiva integral que involucra el contexto biológico, social y ambiental (Macias y col., 2009). La educación alimentaria fue siempre parte del ámbito privado, particularmente familiar. «Sin embargo, la relevancia para la vida tanto del niño como del joven y del adulto, resulta imperiosa que su enseñanza se sistematice dentro de las instituciones educativas» (Más Varela, 2017).

Una proporción muy importante de la vida corresponde a la etapa de adulto y durante esta el estilo de vida saludable es primordial para lograr un envejecimiento exitoso que lleve a una vejez con calidad, y es a través de la EAN que podemos trabajar en mejorar estilos de vida poco saludables.

Por lo anteriormente mencionado, se considera de vital importancia el intercambio a través de diversas instancias educativas, que contemplen la valoración de hábitos saludables permitiendo prolongar años de vida y manteniendo un buen estado de salud.

En esta dirección se proponen como objetivos:

- Describir la propuesta educativa en el EEMPA Juan J. Paso №1305, teniendo como eje la EAN.

- Valorar la propuesta educativa desde la mirada de los jóvenes-adultos. 


\section{Metodología}

El trabajo realizado fue parte de una Práctica de Extensión de Educación Experiencial (PEEE) de la Facultad de Bioquímica y Ciencias Biológicas de la Universidad Nacional del Litoral (FBCB-UNL), que se denominó «Promoción de Educación Alimentaria en Jóvenes y Adultos: desafío conjunto UniversidadComunidad».

El presente trabajo relata la experiencia realizada en la EEMPA Juan J. Paso No1305 de la ciudad de Santa Fe, con 100 alumnos que participaron voluntariamente y que se encontraban cursando el tercer y quinto año durante 2016.

Se realizó un estudio de tipo transversal descriptivo e interpretativo. Se aplicó una encuesta diagnóstica para relevar los hábitos alimentarios de los participantes. Luego, y sobre la base de los resultados de la misma, se diseñaron e implementaron 12 talleres didácticos sobre EAN en jóvenes adultos durante tres semanas consecutivas. Posteriormente, se aplicó la misma encuesta con el fin de poder identificar y comparar los conocimientos y prácticas adquiridas sobre hábitos alimentarios saludables por parte de los alumnos. Por último, se efectuó una encuesta de opinión a los participantes de los talleres para valorar los mismos.

\section{Consideraciones bioéticas}

Esta investigación se realizó en el marco de un proyecto de Práctica de Extensión de Educación Experiencial (PEEE): «Promoción de Educación Alimentaria en Jóvenes y Adultos: desafío conjunto Universidad-Comunidad», aprobado por Resolución Consejo Directivo FBCB N²39/2016 (5).

\section{Resultados}

\section{Primera instancia: encuesta diagnóstica}

Inicialmente, en septiembre del año 2016, se llevó a cabo un primer encuentro en la institución educativa donde se explicó el proyecto a realizar y luego se aplicó una encuesta diagnóstica. El objetivo de la misma fue conocer y describir los hábitos alimentarios de los alumnos de la EEMPA N 1305 de la ciudad de Santa Fe.

\section{Características de la muestra}

De los 100 jóvenes adultos que participaron de la investigación, un $59 \%$ pertenecía al tercer año y el $41 \%$ se encontraba cursando el quinto año. Se observó que la proporción de estudiantes por sexo estuvo representada por un 
$61 \%$ de hombres y un $39 \%$ mujeres. Respecto de la edad, los alumnos tenían entre 18 y 55 años. La mayoría de los alumnos tenía residencia en los distritos norte, este y oeste de la ciudad de Santa Fe, y a su vez la mayoría de ellos (58\%) pertenecía a familias tipo de hasta 4 integrantes, un 42 \% vivía con 5 integrantes o más en su hogar.

En cuanto a los hábitos alimentarios de los jóvenes adultos, un bajo porcentaje de los participantes (32\%) realizaba 4 comidas diarias. Con relación al desayuno, un $56 \%$ de los sujetos no desayunaba diariamente y mencionó que el motivo principal para no realizarlo era levantarse tarde $(50 \%)$ y en menor medida estar inapetente (15\%).

La mayoría de los alumnos (65\%) eligen las infusiones (mate, café, té y mate cocido) para desayunar, y solo un $34 \%$ incluía lácteos. Además, la mayoría (66 \%) mencionó que lo acompañaba con alguna de las siguientes opciones: pan blanco, pan integral, tostadas, galletitas dulces y/o saladas. Cabe destacar que un $96 \%$ de los alumnos consideró importante desayunar. Algunas de las razones que mencionaron fueron:

- «Es la comida más importante del día».

- «Es la primera comida del día que nos da energía».

- «Porque es el primer alimento que nos ayuda a estar bien durante la mañana».

- «Es lo primero que comemos en el día y lo que nos mantiene fuertes».

- Además se vio reflejado el consumo deficitario de algunos grupos de alimentos, como «verduras y frutas», «carnes y huevos», leche, yogur o queso y agua.

\section{Segunda instancia: estrategias didácticas}

\section{Diseño de los talleres desarrollados}

Para la realización de las propuestas didácticas educativas se consideraron como punto de partida los resultados de la encuesta diagnóstica realizada previamente, haciendo hincapié en las problemáticas relacionadas con la alimentación que pudieron ser identificadas en las respuestas de los alumnos.

Con eje en EAN, se llevaron a cabo estrategias lúdico-didácticas integrando la educación para la salud, el consumo y la educación en valores, en el marco de los objetivos y contenidos del currículo de Ciencias Naturales.

La propuesta impulsó la utilización de estrategias que adoptaran un enfoque integral y no la mera transmisión de información en el aula, promocionando y reforzando hábitos saludables, modificando aquellos que sean perjudiciales para 
la salud de todos los jóvenes-adultos, para que sea extendido a las familias y puedan ser un canal para la participación de la comunidad en general.

\section{Implementación de los talleres}

Los talleres se efectuaron durante tres semanas consecutivas en el mes de septiembre de 2016. Se implementaron tres talleres lúdico-didácticos en cuatro cursos de la institución educativa.

\section{Primer taller: "Camino a mi desayuno saludable»}

Para dar inicio al primer taller se mostró un video sobre la importancia del desayuno, sus ventajas y las consecuencias para el cuerpo de no realizarlo. A partir del video, se elaboró una presentación utilizando el programa de acceso libre Prezi, la que se llamó: “Camino a mi desayuno saludable». Contenía recomendaciones y preguntas acerca del desayuno; lo que permitió la participación activa de los alumnos durante el taller. Al finalizar, se entregó a los participantes un crucigrama con frases y preguntas que debían completar sobre el desayuno, pensarlas y responderlas individualmente para luego debatirlas de manera grupal.
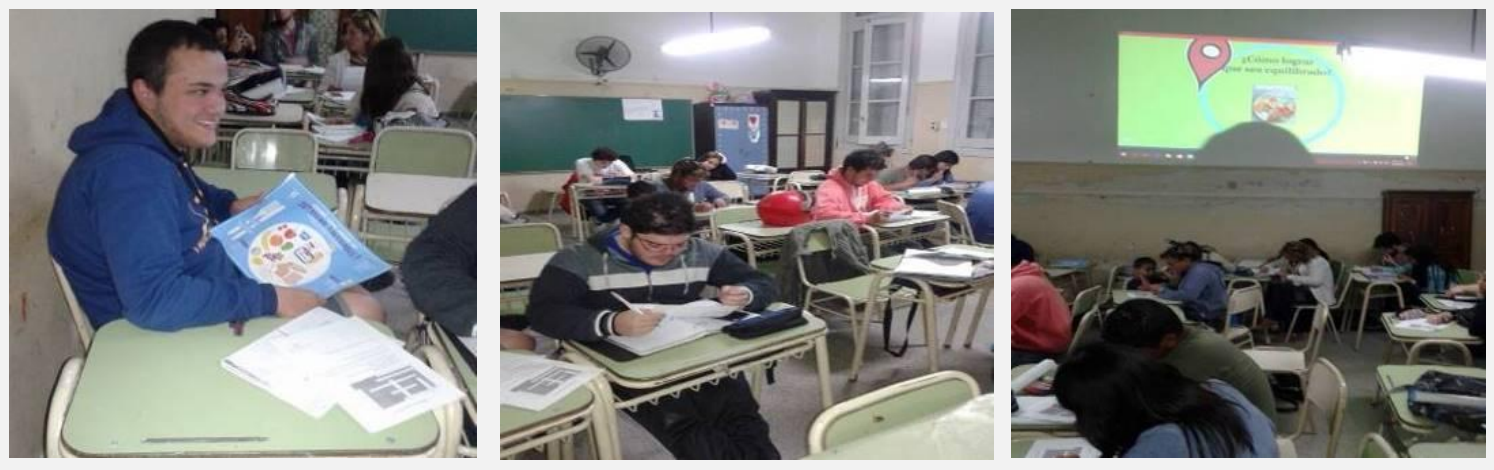

Imagen 1: Alumnos realizando el primer taller. EEMPA Juan J. Paso № 1305. Santa Fe, 2016.

Segundo taller: «El plato nutricional y sus grupos de alimentos»

En este segundo taller, la actividad de inicio fue exhibir el plato nutricional, donde fueron identificando cada grupo de alimentos entre todos los alumnos. Luego se leyeron los mensajes de las GAPA y ellos tenían que ir colocando cada frase donde correspondía el grupo de alimento.

En la actividad de desarrollo se realizó un nutribingo donde, a medida que iban saliendo los alimentos, los tenían que ir colocando en un plato nutricional vacío y ponerlos en el grupo de alimento correspondiente. El juego culminaba 
con el participante que hacía bingo. Fue un juego muy dinámico, participativo donde los alumnos, además de divertirse, aprendieron.

Para finalizar, se propuso un juego disponible en Internet. Los estudiantes tenían que armar un plato saludable con los diferentes grupos de alimentos. Se separaron en dos grupos y el que armaba más platos saludables ganaba el juego. Fue una actividad interactiva e innovadora, donde pudieron integrarse y aprender jugando.
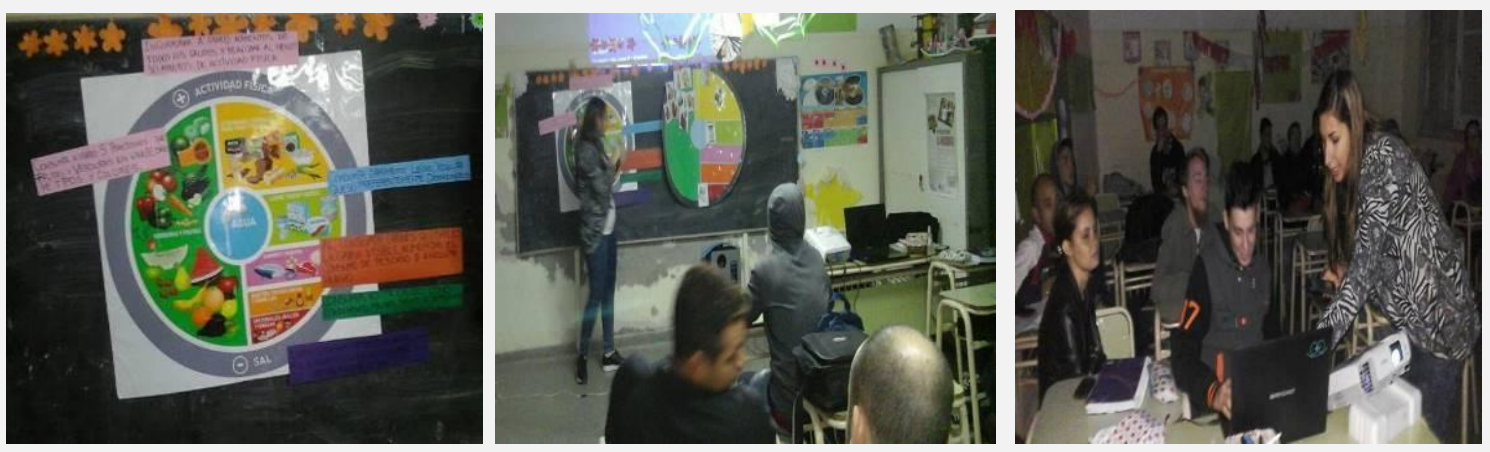

Imagen 2: Alumnos realizando el segundo taller. EEMPA Juan J. Paso n¹305. Santa Fe, 2016.

Tercer taller: «Hidratándonos y ejercitándonos»

El último taller se comenzó con una lluvia de ideas sobre la cantidad de agua que ellos consumían. Luego se realizó una actividad que consistió en ir ubicando vasos de agua en miniatura en un reloj, distribuyéndolos como si fuera un día habitual.

Después se proyectó un video interactivo llamado «8 vasos de agua al día y sus beneficios".

La actividad de desarrollo consistió en un juego con dos cubos grandes con imágenes sobre hidratación y actividad física, y los alumnos los iban tirando al azar. Cuando salía una imagen, se leía la frase y con una manopla que se había repartido a cada uno, ponían el pulgar para arriba si era «verdad» o pulgar para abajo si era «mito». Luego se debatían las respuestas en forma grupal.

Como actividad de cierre se presentaron dos aplicaciones (apps) para que las pudieran utilizar en su celular y en su vida cotidiana aplicando los conocimientos sobre hidratación y actividad física. Nuevamente, se elaboró una presentación utilizando el programa de acceso libre Prezi, se dio la explicación paso por paso acerca de cómo descargar las apps y cómo utilizarlas. En simultáneo, los alumnos iban viendo en sus celulares las apps, probándolas y sacándose dudas, para luego poder usarlas diariamente. 

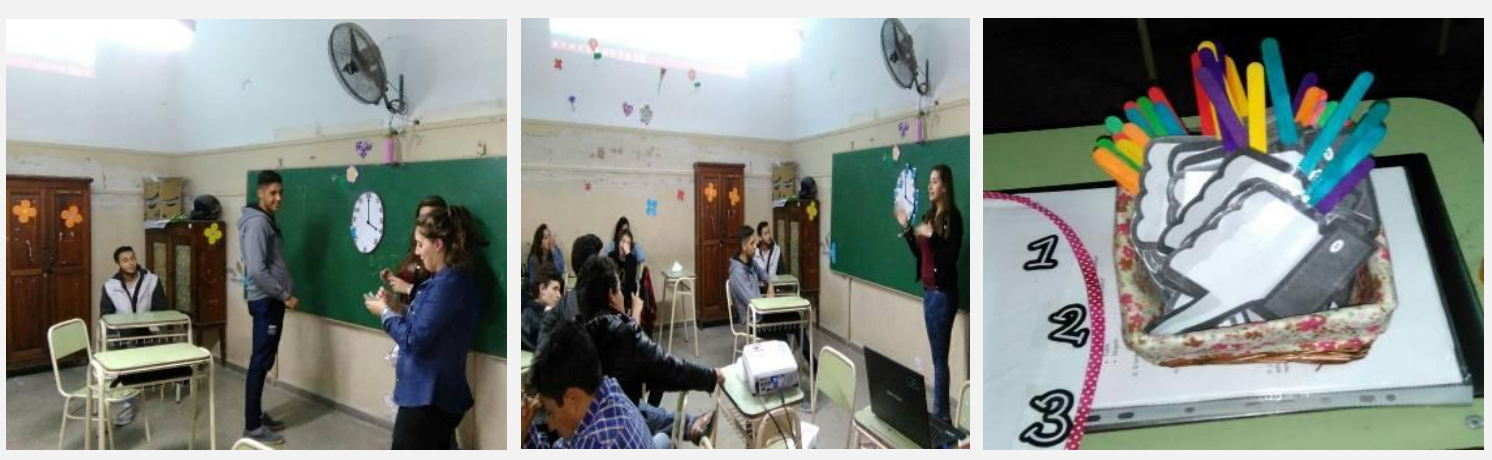

Imagen 3: Alumnos realizando el tercer taller. EEMPA Juan J. Paso №1305. Santa Fe, 2016.

\section{Tercera instancia: prueba de solidez}

Luego de haber transcurrido tres meses de la realización de los talleres en la escuela, se aplicó la prueba de solidez. La misma consistió en repetir la encuesta diagnóstica inicial con el fin de identificar y comparar los conocimientos y prácticas adquiridas sobre hábitos alimentarios saludables por parte de los alumnos.

Al analizar el número de comidas que realizaban diariamente, se observó que más del $50 \%$ de los participantes había aumentado el número de comidas recomendado.

Con respecto a la comida más importante del día, hubo un aumento considerable de los alumnos que comenzaron con el hábito del desayuno.

Se destacó el aumento del consumo de pan blanco, integral y tostadas, y se observó una disminución en el resto de los alimentos ricos en grasas saturadas y azúcares simples. Respecto del consumo recomendado de lácteos, aumentó en la mayoría de los estudiantes.

Con referencia a los grupos de alimentos presentes en la alimentación diaria, los alumnos incrementaron el consumo del grupo "Carnes y huevos», hubo un aumento considerable en los grupos «Verduras y frutas» $y$ «Lácteos»; en menor medida aumentó el consumo del grupo «Legumbres, cereales, papa, pan y pastas», luego de las IEN. Además se vio reflejado el aumento del consumo de agua.

\section{Cuarta instancia: encuesta de opinión}

Esta instancia surgió con el fin de contar con una valoración por parte de los alumnos de los talleres desarrollados. Se indagó sobre la opinión de los participantes acerca de las diferentes actividades llevadas a cabo en este estudio.

Los estudiantes debían calificar los tres talleres desarrollados en la escuela y para ello disponían de una escala de valores de 1 a 5 , donde el 1 representaba 
el menor puntaje y 5 el mayor. El $83 \%$ de los alumnos calificó los talleres desarrollados con el mayor puntaje. Los resultados se pueden ver en el siguiente gráfico.

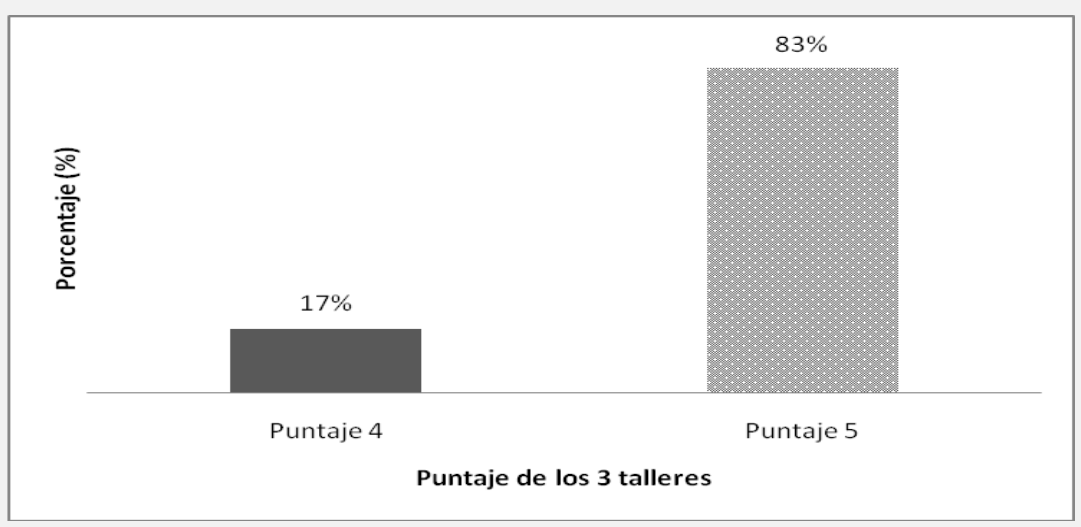

Gráfico 1: Valoración por parte de los alumnos de los talleres realizados.

En la siguiente pregunta debían elegir cuál fue el taller que les resultó más interesante. Más de la mitad de los encuestados (56 \%) respondió que le resultaron interesantes TODOS los talleres realizados en la escuela.

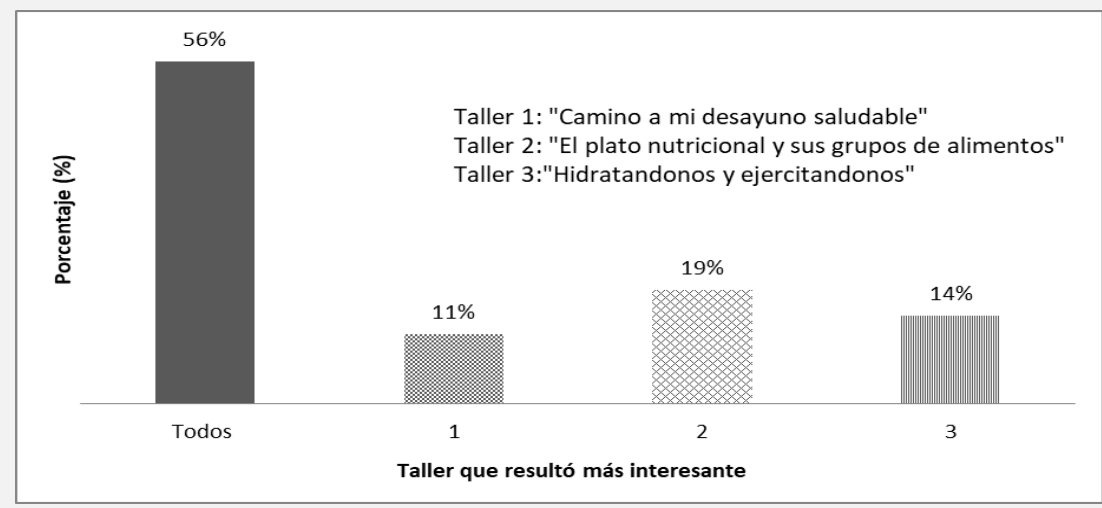

Gráfico 2: Taller desarrollado más interesante para los estudiantes.

Además se recurrió a la identificación y posterior análisis de «frases y/o párrafos significativos», mediante los cuales los participantes expresaron sus consideraciones acerca de los talleres:

1. «Estuvo interesante, me gustó poder expresarme y educarme cada vez mejor». «Los talleres me dejaron enseñanza y reflexión». 
2. «El desayuno es la comida más importante y la alimentación sana es crucial para tener buena salud». «Me enseñó que las frutas son importantes».

3. «La buena alimentación y la actividad física son saludables para nuestra vida».

4. «Me informaron sobre los alimentos y sus beneficios». Cómo, cuándo y qué comer».

5. «Que la gente se eduque y aprenda a conocer su cuerpo, cómo funciona y cómo debe alimentarse y saber lo que come».

6. «Me enseñaron la importancia que la buena nutrición tiene en nuestra vida».

7. «Me gustaron, para poder aprender y saber cómo cuidarme».

8. «Alimentarse bien para un cuerpo saludable». «Buenos hábitos, vida más fácil»

9. «Cuidar nuestra alimentación para tener una buena salud. Muy innovadoras y divertidas».

10. «Alimentarse de forma adecuada y moverse con frecuencia favorece nuestra salud».

De las frases significativas mencionadas por los alumnos se puede decir que para ellos fue positivo e interesante aprender sobre alimentación saludable, la importancia del desayuno, la hidratación y la actividad física. Se ha demostrado que la educación nutricional en el entorno escolar es capaz de influir en los conocimientos sobre alimentación y nutrición.

Asimismo, se les consultó sobre si querían proponer alguna modificación o sugerencia para los talleres realizados, y casi la totalidad de los alumnos opinó que no haría ninguna modificación (93\%). Algunas de las sugerencias hechas por los alumnos que respondieron a esta pregunta (7\%) fueron las siguientes:

1. «Que se dicte un taller donde se vea qué No debemos comer».

2. «Peso y alimentación». 


\section{Discusión}

En la bibliografía consultada no se encuentran datos de investigaciones publicadas que hayan abordado los hábitos alimentarios en jóvenes-adultos en escuelas nocturnas.

Es importante resaltar que la inclusión de los nuevos modos y escenarios de los grupos desfavorecidos y vulnerables, como son la mayoría de los alumnos que acceden y concurren a las escuelas nocturnas, se convierten en una nueva realidad, en una nueva posibilidad y, fundamentalmente, en un nuevo desafío.

Los alumnos de estos centros educativos suelen pertenecer a un nivel socioeconómico bajo, provienen de grupos familiares desarticulados, con un número elevado de hijos, con extensas jornadas laborales. Son jóvenes y adultos que no han podido completar sus estudios por diferentes motivos: enfermedad, trabajo, problemas familiares, económicos, fracasos ante propuestas educativas tradicionales, etc. (Del Río, 2009).

El campo de la educación para jóvenes y adultos se orienta a atender a una población que se caracteriza, por un lado, por no haber podido acceder a la escuela y, por otro lado (en caso de acceder a la escuela), por no haber finalizado los estudios y retomar la escolaridad luego de sucesivos fracasos (Brusilovsky, 2006).

La institución educativa donde se desarrolló la experiencia cuenta con una población de jóvenes adultos proveniente de contextos socioculturales diversos y con características escolares de fracaso.

El aprendizaje en las personas adultas tiene características particulares; ellas son conscientes de sus necesidades educativas, tienen la madurez como para seleccionar los medios y la forma de aprender, si bien por otra parte se asume que los adultos tienen tiempo limitado y deben equilibrar las demandas de la familia, el trabajo y la educación (Ongay y col., 2013).

Se puede afirmar que este estudio mostró que la utilización de actividades lúdicas potencializó el aprendizaje de los alumnos; las estrategias didácticas desarrolladas tuvieron influencia en la construcción de conocimientos sobre alimentación y nutrición. Además, fue posible constatar que hubo modificaciones en las actitudes de los estudiantes, principalmente en la motivación y el interés por la temática abordada, con lo cual varios autores han concordado que los juegos pueden convertirse en instrumentos que posibiliten el aprendizaje significativo por parte de los alumnos (Ausubel y col., 1976).

\section{Reflexiones finales}

Las escuelas nocturnas son instituciones educativas que posibilitan el acceso con equidad al sistema educativo, a jóvenes y adultos que por diversas razones no tuvieron la oportunidad o no han podido concluir el nivel secundario y desean completarlo. 
La EAN y su influencia en la salud requieren contenidos y competencias a trabajar en la escuela con el objetivo de mejorar la conducta alimentaria y elevar la calidad de vida. Si se incorporan estrategias educativas innovadoras, como talleres dinámicos y participativos, se incrementa e incentiva el aprendizaje, por ejemplo, con el uso de juegos.

La EAN utilizando estrategias lúdico-didácticas, es una de las herramientas para proveer una educación más dinámica y participativa, donde los alumnos se involucren individual y grupalmente.

Cuando se potencia la EAN en el ámbito escolar, se puede mejorar directamente la salud y la nutrición de los jóvenes adultos, promoviendo buenos hábitos alimenticios en ellos y en sus familias.

En esta dirección se debe continuar trabajando en facilitar la adopción voluntaria de conductas, comportamientos y elecciones adecuadas en alimentación y nutrición para mantener o mejorar la salud y el bienestar, en la medida en que es una persona activa frente a su realidad, capaz de contribuir al desarrollo propio, al de su familia y de su comunidad.

\section{Agradecimientos}

A la Facultad de Bioquímica y Ciencias Biológicas de la UNL por habernos brindado las herramientas necesarias para llegar a la comunidad.

A la EEMPA Juan J. Paso N¹305 de la ciudad de Santa Fe por habernos abierto sus puertas para desarrollar esta propuesta.

A los alumnos de dicha institución por su compromiso y motivación para colaborar con este proyecto de investigación. 


\section{Referencias bibliográficas}

- $\quad$ Ausubel, D.; Novak, J. y Hanesian, H. (1976). Psicología Educativa: un punto de vista cognoscitivo. Trillas.

- Brusilovsky, S.; Cabrera, M. (2006). Educación escolar de adultos. Noveduc.

- Contento, I.R. (2011). Nutrition Education: Linking Theory, Research, and Practice. En: Garcés, I.C.C. El nutricionista dietista como educador. Jones y Bartlett Publishers. http://escritura.proyectolatin.org/educacion-alimentaria-y-nutricional-en-el-marco-de-laeducacion-para-la-salud/el-nutricionista-como-educador/

- Del Río, A. (2009). La educación a distancia en la educación básica para adultos. Búsquedas y perspectivas. http://repositoriodigital.academica.mx/jspui/bitstream/987654321/21826/1/decisio22_saber4.pdf

- Food and Agriculture Organization/FAO (2017). http://www.fao.org/school-food/en/

- Gil Hernández, A.D.; Maldonado Lozano, J.; Martínez de Victoria Muñoz, E. (2010). Tratado de Nutrición: Nutrición humana en el Estado de Salud (Vol. 3). Editorial Médica Panamericana.

- Macias, A.I.; Quintero, M.L., Camacho, E.J. y col. (2009). La tridimensionalidad del concepto de nutrición: su relación con la educación para la salud. Rev. Chil. Nutr., 36(4), 1129-1135. http://www.scielo.cl/scielo.php?script=sci_arttext\&pid=S071775182009000400010

- Más Varela, S. (2017). Impulsan la educación alimentaria nutricional en escuelas de Santa Fe. http://www.conclusion.com.ar/2017/02/impulsan-la-educacion-alimentaria-nutricional-enescuelas-de-santa-fe/

- Ongay, E.; Saravia, L.; Cortada, D. y col. (2013). Capacitación de funcionarios de servicios de alimentación y nutrición. (LATIn). http://escritura.proyectolatin.org/educacion-alimentariay-nutricional-en-el-marco-de-la-educacion-para-la-salud/capacitacion-de-funcionarios-deservicios-de-alimentacion-y-nutricion/ 